OPEN

SUBJECT AREAS:

SENSORS AND

BIOSENSORS

SYNTHESIS AND PROCESSING

NANOWIRES

NANOSENSORS

Received

26 March 2013

Accepted

15 July 2013

Published

20 January 2014

Correspondence and requests for materials should be addressed to

L.F.F. (luis.fonseca@ upr.edu)

\section{Shape-controlled synthesis of palladium and copper superlattice nanowires for high-stability hydrogen sensors}

\author{
Dachi Yang' ', Jennifer Carpena-Núñez' ${ }^{1}$ Luis F. Fonseca' ${ }^{1}$ Azlin Biaggi-Labiosa² \& Gary W. Hunter ${ }^{2}$
}

\begin{abstract}
'Department of Physics, University of Puerto Rico at Rio Piedras San Juan, PR 00931, USA, ${ }^{2}$ NASA Glenn Research Center, Cleveland, $\mathrm{OH} 44135$, USA.
\end{abstract}

For hydrogen sensors built with pure Pd nanowires, the instabilities causing baseline drifting and temperature-driven sensing behavior are limiting factors when working within a wide temperature range. To enhance the material stability, we have developed superlattice-structured palladium and copper nanowires (PdCu NWs) with random-gapped, screw-threaded, and spiral shapes achieved by wet-chemical approaches. The microstructure of the $\mathrm{PdCu}$ NWs reveals novel superlattices composed of lattice groups structured by four-atomic layers of alternating Pd and $\mathrm{Cu}$. Sensors built with these modified NWs show significantly reduced baseline drifting and lower critical temperature $(259.4 \mathrm{~K}$ and $261 \mathrm{~K}$ depending on the PdCu structure) for the reverse sensing behavior than those with pure Pd NWs (287 K). Moreover, the response and recovery times of the PdCu NWs sensor were of $\sim 9$ and $\sim 7$ times faster than for Pd NWs sensors, respectively.

alladium nanowires (Pd NWs) with large surface-to-volume (S/V) ratio are widely investigated as preferential candidates for hydrogen sensor development. Standardly, palladium absorbs hydrogen to form a hydride (PdHx) that changes its resistivity ${ }^{1-4}$. This behavior can be modified in NWs by the $\alpha-\beta$ phase transition of $\mathrm{PdHx}$ that causes their response to change to a percolation controlled one. Recently, temperaturedependence investigations ${ }^{5}$ within the $120-370 \mathrm{~K}$ range showed that at a failing certain critical temperature the sensing behavior changes from the typical bulk response, where the electrical resistance of the NWs increases, to the percolation-type behavior where the resistance decreases instead. Meanwhile, the $\alpha-\beta$ phase transition ${ }^{2,6,7}$ that occurs above hydrogen $1 \%-2 \%$ volume concentrations ${ }^{8}$, may reduce their stability and reproducibility.

The doping of Pd with other metals has been reported to be an efficient way to reduce or prevent $\alpha-\beta$ phase transitions in $\mathrm{PdHx}$, therefore improving the sensor stability. For example, the transition is reduced when doping with silver (Ag) above 25 atomic\%, and does not occur when the concentration is above 30 atomic $\%$. The doping of $\mathrm{Pd}$ with other materials such as $\mathrm{Cr}$ has also yielded improvements in sensor response and stability ${ }^{10}$. Hence, other Pd-based bimetallic NW $\mathrm{s}^{11-15}$ have been investigated. Composites can form superlattices which are defined as unit cells periodically stacked along a specific direction ${ }^{16}$. For example, the stacking sequence can be “...ABABAB ..."17 or "...ABCABCABC..."18,19. To our knowledge, the investigated superlattice NWs are mostly semiconductors ${ }^{16,17,19-21}$. If the "ABAB..." stacking sequence is applied to Pd-based bimetallic superlattices, Pd atomic layers are thus isolated by those of other metals, which may prevent the $\alpha-\beta$ phase transition. Additionally, it is still a challenge for hydrogen sensors prepared with NWs to further enhance the S/V ratio, even when the diameter of NWs is decreased or Pd nanotubes (NTs) ${ }^{22}$ are employed. For the fabrication of NWs with very small diameter, either costly preparation or complicated procedures are involved ${ }^{23,24}$. In the case of sensors built with Pd NTs, the gas may remain inside the NTs when the hydrogen source disappears thus causing an increase in the sensor's recovery time. So far, few new NW shapes such as spiral-shaped NWs of various materials ${ }^{25-27}$ have been reported. Thus, it is reasonable to propose Pd superlattice-based NWs with desired shapes to enhance the sensor's stability by reducing the $\alpha-\beta$ phase transition of $\mathrm{PdHx}$, as well as the sensor's sensitivity by improving the $\mathrm{S} / \mathrm{V}$ ratio.

Here, superlattices NWs of copper $(\mathrm{Cu})$ and $\mathrm{Pd}(\mathrm{PdCu} \mathrm{NWs})$ with random-gapped, screw-threaded, and spiral shapes have been synthesized by electrochemical deposition and subsequent chemical etching. The enhanced stability of hydrogen sensors built with these new nanostructures is compared with similar data using Pd nanowires and the results discussed. 


\section{Results}

The PdCu NWs were synthesized by electrochemically depositing $\mathrm{PdCu}$ NWs with pre-contoured shapes inside the nanochannels of anodic aluminum oxide (AAO) templates, and then partially etching away the copper. The synthesis process and the resultant products are described in Figure 1a and 1b, respectively.

PdCu NWs pre-contoured shapes before etching. The representative field-emission scanning electron microscope (SEM) images of $\mathrm{PdCu}$ NWs in Figure $2 \mathrm{a}$ are seen to be uniform $(\sim 70 \mathrm{~nm}$ in diameter), from which the periodic-gapped contours are observed on the surface. More SEM characterizations provided in the Supplementary material (Supplementary Figure S1) show other contours. Correspondingly, the TEM images reveal that the surface shapes of NWs can be smooth (Figure 2b), random-chapped (Figure 2c) and periodicgapped (Figure $2 \mathrm{~d}-\mathrm{e}$ ) as designed. A representative energy dispersive X-ray spectroscopy (EDS) mapping was done to confirm the composition (Supplementary Figure S2).

PdCu NWs with modified shapes after etching. The SEM image in Figure 3a shows screw-threaded PdCu NWs, from which the NWs arrays have similar diameter $(65 \mathrm{~nm})$. Correspondingly, the presence of the PdCu NW with random-chapped (Figure 3b), random-gapped (Figure 3c), screw-threaded (Figure 3d) and spiral (Figure 3e) shapes were confirmed with TEM observations, respectively. More SEM characterizations (Supplementary Figure S3) can be seen with other modified shapes. We tried various conditions, and found that the desired shapes were achieved according to the contours of pre-synthesized PdCu NWs, etching temperature and duration. For example, random-chapped (Figure 3b), random-gapped (Figure 3c), screw-threaded (Figure $3 \mathrm{~d}$ ), and spiral (Figure $3 \mathrm{e}$ ) NWs $(\sim 70 \mathrm{~nm}$ in diameter) were achieved by etching for $20 \mathrm{~min}, 25 \mathrm{~min}, 15 \mathrm{~min}$, and 25-35 min at $50^{\circ} \mathrm{C}$, respectively. If excessive etching was applied, broken PdCu NWs were obtained (Supplementary Figure S4).

Observation of the superlattice structure of PdCu NWs. A TEM image of PdCu NWs (Figure 4a) with smooth surface was analyzed before the etching procedure. From the selected area electron diffraction (SAED) pattern in Figure $4 \mathrm{~b}$ and the high-resolution TEM (HR-TEM) in Figure 4c, we can conclude that PdCu NWs show high crystalline quality. By further magnification, we can see two $\mathrm{Cu}$ and two Pd lattice planes (both (111) planes) forming one $\mathrm{PdCu}$ group, with interplanar spacing varying between $\sim 0.55 \mathrm{~nm}$ (Figure $4 \mathrm{~d}$ ), $\sim 0.60 \mathrm{~nm}$ (Figure $4 \mathrm{e}$ ) and $\sim 0.65 \mathrm{~nm}$ (Figure $4 \mathrm{f}$ ) as the

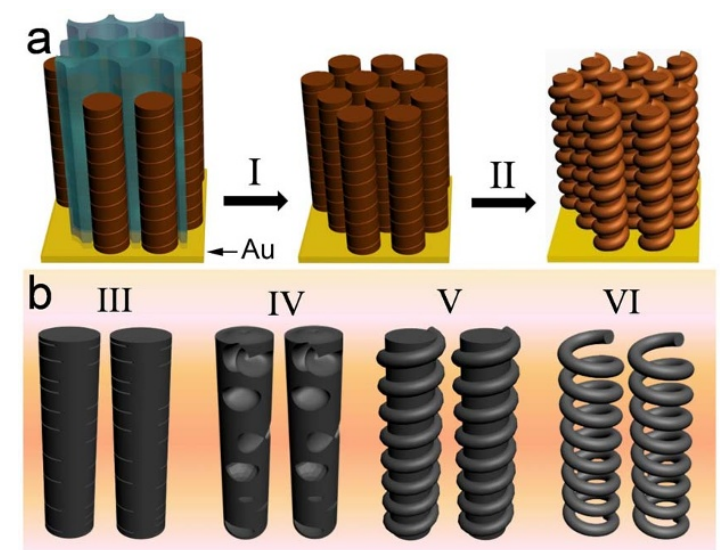

Figure 1 | Synthesis process and resultant products. Panel (a), schematic synthesis of shapes-modified superlattice PdCu NWs. I, preelectrodeposited PdCu NWs released by dissolving AAO template. II, partially etching $\mathrm{Cu}$. Panel (b), the resultant products obtained with III random-chapped, IV random-gapped, V screw-threaded and VI spiral shapes.

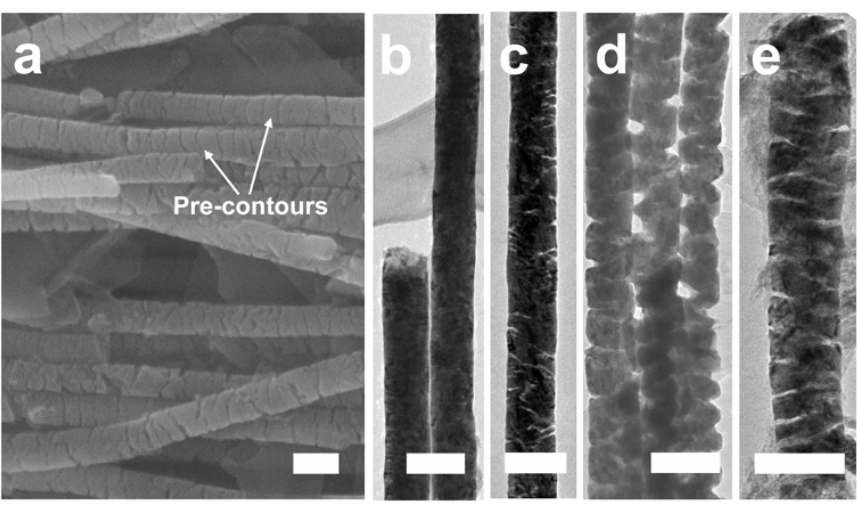

Figure $2 \mid$ The pre-contoured shapes. The SEM images of PdCu NWs arrays with the shapes of (a) periodic-gapped shapes; The TEM images with (b) smooth, (c) random-chapped and (d-e) periodic-gapped shapes. The scale bars are all $100 \mathrm{~nm}$.

result of the lattices coincidence between $\mathrm{Pd}$ and $\mathrm{Cu}$. The periodic groups arrays (...AAAA...sequence) constitutes a superlattice, as is schematically depicted in Figure $4 \mathrm{~g}$. Moreover, a twin structure (Supplementary Figure S5a) was observed, where the twin boundary is composed of coherent $\mathrm{PdCu}$ superlattices groups. Additionally, the interface between two Pd lattices and one PdCu lattice group was resolved along the right line of the inset TEM image in Figure S5b of the Supplementary information, which further confirms that one PdCu lattice group is comprised of two $\mathrm{Pd}$ and two $\mathrm{Cu}$ lattices. Additionally, we also resolved $\mathrm{PdCu} \mathrm{NW}$ with modified shapes, and found that the superlattice microstructures were not destroyed by etching (Supplementary Figure S6).

Sensor prototypes and sensing response within 150-370 K temperature range. To build hydrogen sensors with these new materials, inter-digitated electrodes (IDEs) were employed to integrate the $\mathrm{PdCu} \mathrm{NWs}{ }^{5}$. The sensor prototypes in Figure 5a were built with multiple NWs, in which PdCu NWs across the IDEs are randomly aligned and overlapped (Supplementary Figure S7). In this way, $\mathrm{PdCu}$ NWs with shapes mentioned above can be integrated as desired. Here we take PdCu NWs with screw-threaded shape as an example for description. A SEM image is presented in Figure S8 (top panel, Supplementary information), from which the $\mathrm{Cu}$ atomic composition obtained from EDS analysis is $\sim 31 \%$. Figure $5 \mathrm{~b}$ shows a representative sensing response tested at $310.3 \mathrm{~K}$, in which one can see that with an applied voltage of $0.15 \mathrm{~V}$, a hydrogen concentration as low as $0.1 \%(\mathrm{v} / \mathrm{v})$ can be detected. Similarly, other sensors built
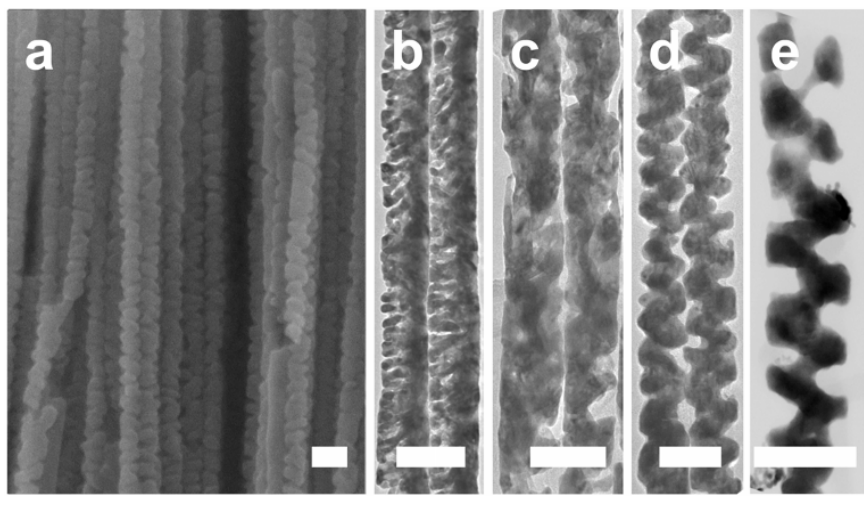

Figure $3 \mid$ The modified shapes. The SEM images of PdCu NWs arrays with (a) screw-threaded shapes. The TEM images of PdCu NWs with (b) random-chapped, (c) random-gapped, (d) screw-threaded and (e) spiralshaped shapes. The scale bars are all $100 \mathrm{~nm}$. 

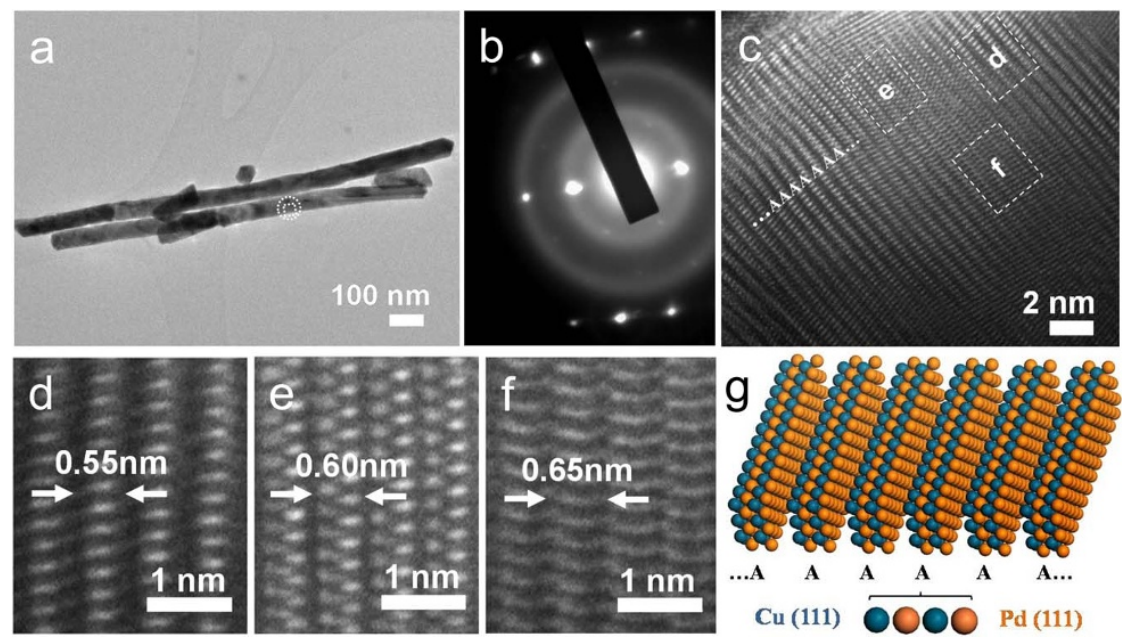

Figure $4 \mid$ Observation of the superlattice of PdCu NWs. (a), The TEM image of a PdCu NWs. (b), The SAED pattern taken from the dashed circle in (a). (c), A HR-TEM image taken from the dashed rectangle in (a), the enlarged HR-TEM images in (d), (e) and (f) are from the corresponding dashed box (d), (e) and (f). (g), The scheme of the superlattice.

with NWs of smooth (central panel, Supplementary Figure S8), and random-gapped (bottom panel, Supplementary Figure S8) shapes were studied. However, either a non-linear relation between the change in the electrical resistance and the applied hydrogen concentration was obtained (central panel, Supplementary Figure S8) or a higher temperature $(370 \mathrm{~K})$ was required to get a better response (bottom panel, Supplementary Figure S8). The temperature stability of the screw-threaded NWs was tested within 150-370 K range (For detail, see Supplementary Figure S9). We found that at higher temperatures (e.g., at $310 \mathrm{~K}$ ), the sensor shows a response with an increase in resistance $(\mathrm{RH}(+))$, while on at lower temperatures (e.g., at $150 \mathrm{~K}$ ), it responds with a decrease in resistance (RH (-)). However, when the temperature reached to $259.3 \mathrm{~K}$, weak responses were detected even if $3 \% \mathrm{H}_{2}(\mathrm{v} / \mathrm{v})$ was introduced. Accordingly, the temperature dependent resistance change $(\Delta \mathrm{R})$ plotted in Figure $5 \mathrm{c}$ indicates that the critical temperature where the sensing behavior is reversed occurs at $\sim 259.4 \mathrm{~K}$, which is much lower than the values observed in Pd NWs systems $(287 \mathrm{~K})^{5}$. Similarly, the sensors with NWs of random-gapped shapes were investigated, and the critical temperature in Figure $5 \mathrm{~d}$ was obtained $\sim 261 \mathrm{~K}$ (For detail, see Supplementary Figure S10), which is close to that of the screw-threaded shapes $(\sim 259.4 \mathrm{~K})$.
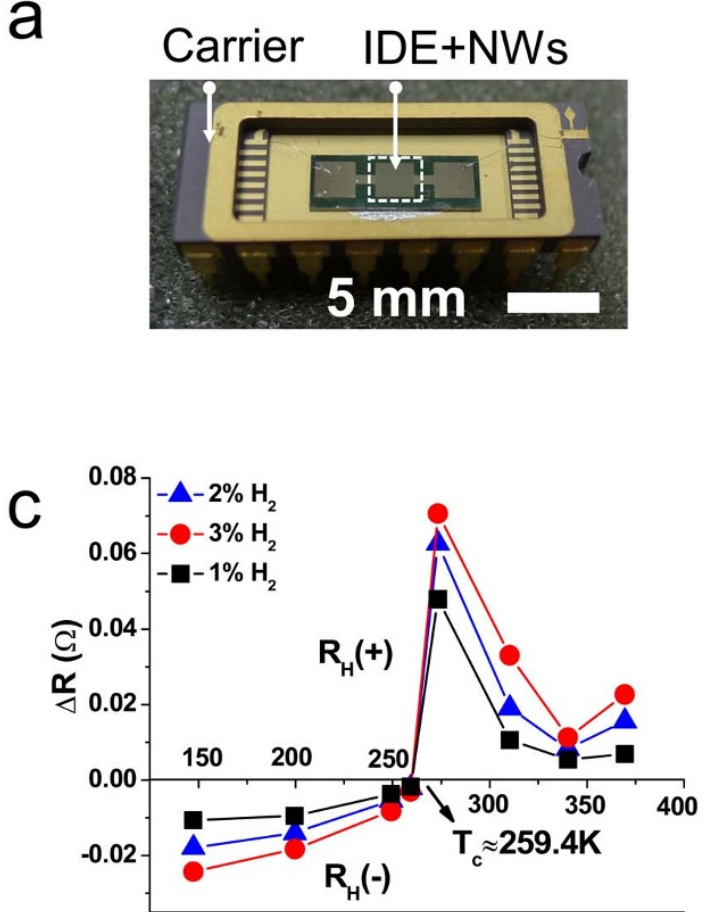

Temperature (K)
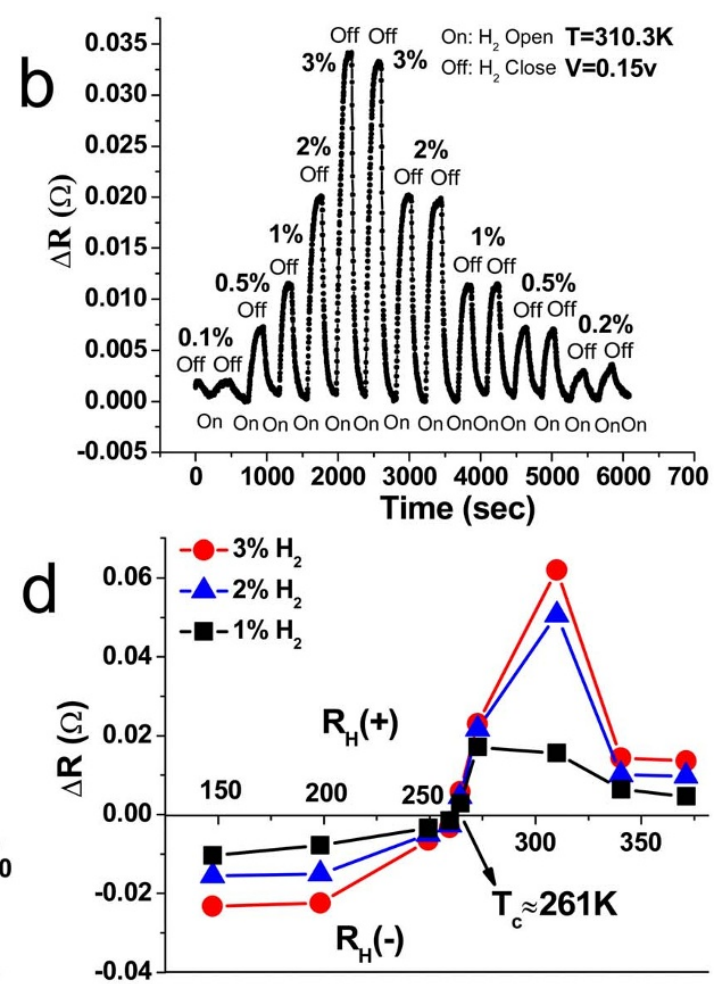

Temperature (K)

Figure $5 \mid$ Hydrogen sensor prototype and sensing response. (a), The hydrogen prototype. (b), The $\Delta \mathrm{R}$ response plots of NWs with screw-threaded shapes at $310.3 \mathrm{~K}$. The temperature-dependence $\Delta \mathrm{R}$ plots of (c) screw-threaded and (d) random-gapped PdCu NWs. The resistance modes, negative values (below Tc) corresponds to the $\mathrm{RH}(-)$ mode and positive ones to the $\mathrm{RH}(+)$ mode. 
Additionally, to investigate the baseline stability of the sensors, we compared PdCu NWs sensors against Pd NWs ones built in similar ways. Pristine sensors were exposed to a $2000 \mathrm{sccm}$ gas flow of $100 \%$ $\mathrm{N}_{2}$ and $0.5 \% \mathrm{H}_{2} / 99.5 \% \mathrm{~N}_{2}(\mathrm{v} / \mathrm{v})$ alternating every minute for 200 minutes and the baseline stabilization was analyzed (Figure 6a). The comparison shows that the $\mathrm{PdCu}$-based sensor achieves a stable baseline significantly faster than the Pd-based one. From further analysis (Figure $6 \mathrm{~b}-\mathrm{c}$ ), we found that the response and the recovery times of the PdCu NWs sensor was of $\sim 9$ and $\sim 7$ times faster than those of the Pd NWs sensors, respectively. However, with the same applied voltage, the sensitivity of the sensors with Pd NWs is of $\sim 4$ times larger than that with $\mathrm{PdCu} \mathrm{NWs}$. These results are consistent with the fact that the PdCu NWs still contain Pd domains.

\section{Discussion}

When samples were prepared before etching, the electrochemical deposition of pre-contoured NWs plays a crucial role in the preparation of PdCu NWs with textured surfaces. In our electrochemical deposition, we tried various electrolytes and parameters, finding that the contours of PdCu NWs were highly dependent on the Pd and $\mathrm{Cu}$ cations relative concentrations, $\mathrm{pH}$ value of the electrolytes, and applied voltages. For example, PdCu NWs with a smooth surface (Figure $2 \mathrm{~b}$ ) formed at the conditions \{voltage $1.2 \sim 2.2 \mathrm{~V}, \mathrm{Pd}: \mathrm{Cu}$ cations molar content $1 \sim 1.5$ and $\mathrm{pH} 7 \sim 8\}$. In this case both, Pd and $\mathrm{Cu}$ cations, are evenly electrodeposited forming superlattice NWs structures. While $\mathrm{PdCu}$ NWs with random-gapped (Supplementary Figure 2), random-chapped (Figure 2c) and periodic-gapped (Figure $2 \mathrm{~d}-\mathrm{e}$ ) contours were usually obtained at the conditions \{voltage $1.3 \sim 1.8 \mathrm{~V}, \mathrm{Pd}: \mathrm{Cu}$ cations molar content 0.9 and $\mathrm{pH} 5 \sim 6\}$, \{voltage $2 \sim 2.8 \mathrm{~V}, \mathrm{Pd}:$ Cu cations molar content $1 \sim$ 2 and $\mathrm{pH} 7 \sim 9$, and \{voltage $1.4 \sim 2.6 \mathrm{~V}, \mathrm{Pd}: \mathrm{Cu}$ cations molar content 1 and $\mathrm{pH} 4 \sim 6$, respectively. It has been reported that the enriched $\mathrm{H}^{+}$ions in an acidic solution around the AAO-channel wall produces a higher content of adsorbed hydrogen, which allows the $\mathrm{Pd}$ cations to be deposited along the wall in the presence of the $\left[\mathrm{PdCl}_{\mathrm{n}}\right]^{(\mathrm{n}-2)-}(2 \leq \mathrm{n} \leq 4)$ while the $\mathrm{Cu}$ ones are not ${ }^{25,28}$. We should point out that $\mathrm{Pd}\left(\mathrm{NH}_{3}\right)_{4} \mathrm{Cl}_{2}$ rather than $\mathrm{PdCl}_{2}$ was selected for the electrolyte, otherwise $\mathrm{Pd}$ nanosprings would be obtained after etching $^{25}$. Additionally, an appropriate voltage was applied to guarantee that $\mathrm{Cu}$ cations were able to be electrodeposited in-between the Pd atoms and thus form PdCu superlattices.

For the PdCu NWs synthesized here, $\mathrm{Cu}$ ions are co-electrodeposited alternately with Pd ones rather than being separated as it is in the case of Pd nanosprings that form after completely etching away the $\mathrm{Cu}$ atoms ${ }^{25}$. In order to partially etch $\mathrm{Cu}$ ions away from as-synthesized $\mathrm{PdCu} \mathrm{NWs}$, mixed solutions of $\mathrm{CuCl}_{2}$ and $\mathrm{HCl}$ were utilized.

Twining superlattices have been reported previously ${ }^{16,19,20}$, which are usually featured in semiconductors based on a periodic array of twinned stacking faults. However, in the case of PdCu NWs, both the $\mathrm{Pd}$ and $\mathrm{Cu}$ are metallic and face-centered. The (111) lattice spacing of $\mathrm{Pd}$ and $\mathrm{Cu}$ are $0.22 \mathrm{~nm}$ and $0.20 \mathrm{~nm}$, respectively, and thus the distortion between $\mathrm{Cu}$ and $\mathrm{Pd}$ planes leads to lattice groups different in width (Figure $4 \mathrm{~d}-\mathrm{f}$ ). In our case, four layers of alternating Pd and $\mathrm{Cu}$ atomic planes form a $\mathrm{PdCu}$ group, and then $\mathrm{PdCu}$ groups' arrays constitute a superlattice (Figure $3 \mathrm{~d}$ ). In other cases, superlattices were reported with the atomic planes stacked layer by layer ${ }^{17,19}$.

Our PdCu superlattice structures are made of four alternating layers of $\mathrm{Pd}$ and $\mathrm{Cu}$ atoms, as shown in Figure 4. In such case, $\mathrm{Pd}$ atomic layers were isolated by $\mathrm{Cu}$ atoms, and the $\alpha$ - $\beta$ phase transition is expected to be reduced or prevented depending on the fractional percentage of $\mathrm{Cu}$. The $\mathrm{Cu}$ atomic fraction of the $\mathrm{PdCu}$ NWs in the top-panel, central-panel and bottom-panel (Supplementary Figure S8) are $31 \%, 2 \%$ and $44 \%$, respectively. However, PdCu NWs with screw-thread shapes corresponding to $31 \%$, show the best stability when compared with the other two. On one hand, the observation confirms that $\mathrm{PdCu}$ NWs show a better response than $\mathrm{Pd}$ NWs with very low $\mathrm{Cu}$ concentration (as in the case of $2 \%$ ) do. On the other hand, the sample with a $44 \% \mathrm{Cu}$ average fraction was not modified by etching away the excess $\mathrm{Cu}$ that apparently prevents a faster absorption of hydrogen in some regions of the NW. Thus, the etching process is important for the sensing response.

Changes in the mechanical properties of PdCu and Pd NWs due to hydrogen absorption were used as another way to test the changes in the crystal structure due to the $\mathrm{H}$-absorption. Observations were made by performing in-situ TEM-AFM tests on pristine and hydrogen-exposed Pd and PdCu NWs under axial compression and tension. From the stress-displacement analysis, pristine-to-hydride approximate embrittlement ratios of $1: 5$ and $1: 4$ were obtained for the Pd NWs and PdCu NWs, respectively. Such results confirm that the $\mathrm{PdCu}$ structure expands less by the insertion of $\mathrm{H}$ atoms than the Pd one. The detailed description can be found in Supplementary Figure S11-S12.

Our results are consistent with NWs made of $\mathrm{PdCu}$ superlattices in which the $\mathrm{PdH}_{\mathrm{x}}$ phase transition that occurs in Pd during the hydrogen absorption cycle is prevented by the incorporation of the $\mathrm{Cu}$ atoms. Thus, the volume expansion is reduced producing lesser baseline instability and embrittlement. As mentioned above, our experiments suggest that Pd domains are still present that contribute to the saturation curve of the sensor baseline. The larger surface to volume ratios found in the screw-threaded and spiral shapes contribute to the higher sensitivities and the shorter diffusion paths to the significantly faster response times measured in the PdCu sensors.

In summary, $\mathrm{PdCu}$ superlattice $\mathrm{NWs}$ with desired randomgapped, spiral, and screw threaded shapes have been achieved by wet-chemical routes. The technique may serve as a general approach to synthesize composite superlattice NWs of Pd and other metals
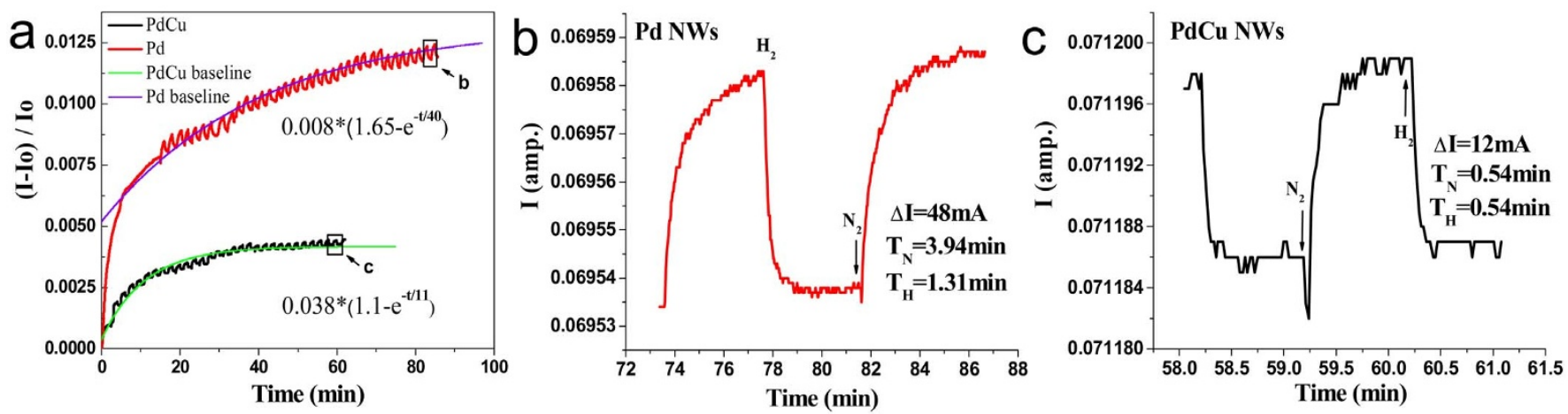

Figure 6 The baseline and response comparison between the Pd NWs and PdCu NWs under the same testing conditions. (a), The baseline analysis of $\mathrm{PdCu}$ and $\mathrm{Pd} \mathrm{NWs}$ under $\mathrm{H}_{2}$ flux $(0.5 \%$ (v/v) at room temperature and atmospheric pressure. The enlarged current versus time plots from

(a) for on-off events showing the response and recovery behaviors of (b) Pd NWs and (c) PdCu NWs. 
Table 1 | Electrodeposition parameters. PdCu NWs with pre-contoured shapes were electrodeposited inside the nanochannel ( $\sim 70 \mathrm{~nm}$ in diameter) of the AAO under various $\mathrm{Pd}$ : $\mathrm{Cu}$ cations molar ratio and $\mathrm{pH}$ value of the electrolyte, and applied voltage

\begin{tabular}{lccc} 
Shapes of PdCu NWs & $\begin{array}{c}\mathrm{Pd} \text { : Cu cations } \\
\text { molar ratio }\end{array}$ & $\begin{array}{c}\text { Electrolyte } \\
\mathrm{pH}\end{array}$ & $\begin{array}{c}\text { Applied voltage } \\
\text { (volt) }\end{array}$ \\
\hline Smooth & $1 \sim 1.5$ & $7 \sim 8$ & $1.2 \sim 2.2$ \\
Random-gapped & 0.9 & $5 \sim 6$ & $1.3 \sim 1.8$ \\
Random-chapped & $1 \sim 2$ & $7 \sim 9$ & $2.0 \sim 2.8$ \\
Periodic-gapped & 1 & $4 \sim 6$ & $1.4 \sim 2.6$ \\
\hline
\end{tabular}

with modified shapes to be used in advanced sensor devices and catalysis. Hydrogen sensor prototypes built with screw-threaded and random-gapped shapes showed better stability than those with pure Pd NWs. The response time and the recovery time of the $\mathrm{PdCu}$ NWs sensor were of $\sim 9$ and $\sim 7$ times faster than those of the Pd NWs ones, respectively. The temperature-stability studies provide an experimental base for hydrogen sensors systems that work in a wide range of temperatures such as those in aerospace crafts working in low temperature environments. Our shaped-modified $\mathrm{PdCu}$ superlattice NWs may also have potential in high-efficiency catalyst application.

\section{Methods}

Samples preparation. The AAO templates were fabricated similarly to previously reported routines ${ }^{29,30}$. The electrolyte for $\mathrm{NW}$ electrodeposition contains $1 \mathrm{~g} \mathrm{Pd}$ $\left(\mathrm{NH}_{3}\right)_{4} \mathrm{Cl}_{2}, 3 \mathrm{~g} \mathrm{Na}_{3} \mathrm{C}_{6} \mathrm{H}_{5} \mathrm{O}_{7} 2 \mathrm{H}_{2} \mathrm{O}, 0.5 \sim 1.15 \mathrm{~g} \mathrm{CuSO}_{4} \cdot 5 \mathrm{H}_{2} \mathrm{O}$ and $0.55 \mathrm{~g} \mathrm{H}_{3} \mathrm{BO}_{3}$. The electrolyte was buffered to $\mathrm{pH} 2 \sim 10$ with $\mathrm{HCl}$ or $\mathrm{NaOH}$ solution. A thick Au layer was sputtered onto one planar surface side of the AAO templates, fully blocking the pores, to serve as the working electrode. With a graphite plate as the counter electrode, the PdCu NWs were electrodeposited inside the nanochannels of AAO template at room temperature, and the electrodeposition parameters and the applied electrolyte were summarized in Table 1. The as-synthesized samples were dissolved in $1 \mathrm{M}$ $\mathrm{NaOH}$ solution, and then rinsed in deionized water thoroughly.

The pre-contoured NWs were immersed in a solution of $0.3 \mathrm{M} \mathrm{CuCl}_{2}$ with $\mathrm{pH} 3$ (tuned with $\mathrm{HCl}$ ), $\mathrm{PdCu} \mathrm{NWs}$ with random-chapped, random-gapped, screwthreaded, and spiral shapes were achieved by etching for $20 \mathrm{~min}, 25 \mathrm{~min}, 15 \mathrm{~min}$, and $25-35 \mathrm{~min}$ at $50^{\circ} \mathrm{C}$, respectively. Finally the samples were thoroughly rinsed.

Microstructural characterizations. After the samples dried, the PdCu NWs were characterized by using SEM (JEOL JSM-7500 F, at $2 \mathrm{KV}$ ) with EDS (OXFORD), TEM (Zeiss LEO 922, at $200 \mathrm{KV}$ ) and HR-TEM (JEOL-2200, at $200 \mathrm{KV}$ ).

Building sensors and testing the $\mathrm{H}_{2}$ sensing response. In order to integrate the NWs to the resistive sensors, IDE platforms were utilized. The NWs were transferred by first sonicating the bundled NWs in alcohol and then dripping the suspension onto the electrodes arrays; the mounting of the IDE on a chip carrier platform was made by bonding the IDE to aluminum wires using a wire bonder (Kulicke \& Soffa 4700). The sensing measurement routine was similar to our previous work ${ }^{5}$. The testing chamber consisted of a cryostat cooled with liquid nitrogen where the temperature was kept constant for $20 \mathrm{~min}$ using a temperature controller (Lake shore, Model 330), with a temperature stability of the order of $\pm 0.2 \mathrm{~K}$. In all cases, a mixed flow of $\operatorname{Ar}(99.999 \%)$ and $\mathrm{H}_{2}(99.9999 \%)$ was used to fill the chamber and the $\mathrm{H}_{2}$ concentration was controlled using gas flow controllers (MKS MASS-FLOW). Initial stabilization, prior to the measurements, was obtained by flowing $1 \% \mathrm{H}_{2}$ (volume)/ $\mathrm{Ar}$ (volume) gas mixture until the electrical current at a constant voltage became stable. The electrical voltage was applied with a computer-controlled picoammeter (Keithley, model 6487) and the electrical current was recorded as a function of time.

1. Ramgir, N. S., Yang, Y. \& Zacharias, M. Nanowire-Based Sensors. small 6, 1705-1722 (2010).

2. Favier, F., Walter, E. C., Zach, M. P., Benter, T. \& Penner, R. M. Hydrogen sensors and switches from electrodeposited palladium mesowire arrays. Science 293, 2227-2231 (2001)

3. Yang, F., Kung, S. C., Taggart, D. K. \& Penner, R. M. Hydrogen Sensing with a Single Palladium Nanowire. Sens. Lett. 8, 534-538 (2010).

4. Offermans, P. et al. Ultralow-power hydrogen sensing with single palladium nanowires. Appl. Phys. Lett. 94, 223110 (2009).

5. Yang, D. et al. Temperature-Activated Reverse Sensing Behavior of Pd Nanowire Hydrogen Sensors. small 9, 188-192 (2012).

6. Yang, F., Taggart, D. K. \& Penner, R. M. Joule heating a palladium nanowire sensor for accelerated response and recovery to hydrogen gas. small 6, 1422-1429 (2010).
7. Li, H., Xu, H. \& Li, W. Study of $\mathrm{n}$ value and alpha/beta palladium hydride phase transition within the ultra-thin palladium composite membrane. J. Membr. Sci. 324, 44-49 (2008).

8. Yang, F., Taggart, D. K. \& Penner, R. M. Fast, Sensitive Hydrogen Gas Detection Using Single Palladium Nanowires That Resist Fracture. Nano Lett. 9, 2177-2182 (2009).

9. Makrides, A. Absorption of Hydrogen by Silver-Palladium Alloys. J. Phys. Chem. 68, 2160-2169 (1964)

10. Hunter, G. W., Xu, J. C. \& Makel, D. B. Case Studies in Chemical Sensor Development. BioNanoFluidic MEMS. 8, 197-231 (2008).

11. Jo, S. Y., Kang, B. R., Kim, J. T., Ra, H. W. \& Im, Y. H. The synthesis of single PdAu bimetallic nanowire: feasibility study for hydrogen sensing. Nanotechnology 21, 055604 (2010).

12. Zeng, X.-Q. et al. Networks of Ultrasmall Pd/Cr Nanowires as High Performance Hydrogen Sensors. Acs Nano 5, 7443-7452 (2011).

13. Xiao, Y. K., Yu, G., Yuan, J., Wang, J. Y. \& Chen, Z. Z. Fabrication of Pd-Ni alloy nanowire arrays on HOPG surface by electrodeposition. Electrochim. Acta 51, 4218-4227 (2006).

14. Xu, C. L., Li, H., Xue, T. \& Li, H. L. Fabrication of CoPd alloy nanowire arrays on an anodic aluminum oxide/Ti/Si substrate and their enhanced magnetic properties. Scr. Mater. 54, 1605-1609 (2006).

15. Tasaltin, N., Ozturk, S., Kilinc, N., Yuzer, H. \& Ozturk, Z. Z. Fabrication of Pd-Fe nanowires with a high aspect ratio by AAO template-assisted electrodeposition. J. Alloys Compd. 509, 3894-3898 (2011).

16. Xiong, Q., Wang, J. \& Eklund, P. Coherent twinning phenomena: Towards twinning superlattices in III-V semiconducting nanowires. Nano Lett. 6 , 2736-2742 (2006).

17. Gudiksen, M. S., Lauhon, L. J., Wang, J., Smith, D. C. \& Lieber, C. M. Growth of nanowire superlattice structures for nanoscale photonics and electronics. Nature 415, 617-620 (2002).

18. Wang, J. G., Tian, M. L., Mallouk, T. E. \& Chan, M. H. W. Microtwinning in template-synthesized single-crystal metal nanowires. J. Phys. Chem. B 108, 841-845 (2004).

19. Caroff, P. et al. Controlled polytypic and twin-plane superlattices in III-V nanowires. Nat. Nanotechnol. 4, 50-55 (2009).

20. Algra, R. E. et al. Twinning superlattices in indium phosphide nanowires. Nature 456, 369-372 (2008).

21. Yoo, B. et al. Electrodeposition of thermoelectric superlattice nanowires. $A d v$. Mater. 19, 296-299 (2007).

22. Mohl, M. et al. Formation of CuPd and CuPt Bimetallic Nanotubes by Galvanic Replacement Reaction. J. Phys. Chem. C 115, 9403-9409 (2011).

23. Zhang, Z., Li, M., Wu, Z. \& Li, W. Ultra-thin PtFe-nanowires as durable electrocatalysts for fuel cells. Nanotechnology 22, 015602 (2011).

24. Zhang, Z. X. et al. Growth of ultrafine ZnS nanowires. Nanotechnology 18, 145607 (2007).

25. Liu, L., Yoo, S.-H., Lee, S. A. \& Park, S. Wet-Chemical Synthesis of Palladium Nanosprings. Nano Lett. 11, 3979-3982 (2011).

26. Mcllroy, D. N., Zhang, D., Kranov, Y. \& Norton, M. G. Nanosprings. Appl. Phys. Lett. 79, 1540-1542 (2001).

27. Bae, S. Y., Lee, J., Jung, H., Park, J. \& Ahn, J. P. Helical structure of singlecrystalline ZnGa2O4 nanowires. J. Am. Chem. Soc. 127, 10802-10803 (2005).

28. Liu, L. \& Park, S. Direct Formation of Thin-Walled Palladium Nanotubes in Nanochannels under an Electrical Potential. Chem. Mater. 23, 1456-1460 (2011).

29. Masuda, H. \& Fukuda, K. Ordered metal nanohole arrays made by a two-step replication of honeycomb structures of anodic alumina. Science 268, 1466-1468 (1995).

30. Meng, G. et al. Ordered Ni nanowire tip arrays sticking out of the anodic aluminum oxide template. J. Appl. Phys. 97, 064303 (2005).

\section{Acknowledgments}

This work was financially supported by NASA URC (Grant NNX08BA48A). Access to the Nanoscopy Facility of the Institute for Functional Nanomaterials at UPR (NSF Grant $\# 1002410)$ is acknowledged. The GRC NASA Summer Faculty Program provided a method to pursue this work, resulting in valuable discussions and the use of the Chemical Sensor Characterization Facility. The authors thank Mr. Luis Valentín for usage of sensor testing facilities and Mr. Oscar Resto for the helpful TEM characterization.

\section{Author contributions}

D.Y. conceived the synthesis experiments, carried out microstructural analysis, and the hydrogen sensing test under diverse temperatures. D.Y. and L.F. wrote the paper. L.F., A.B. and G.H. tested the samples of pure Pd NW and PdCu NWs under $0.5 \%$ hydrogen (v/v). J.C. performed the HR-TEM characterization and mechanical test. All authors discussed the results and commented on the manuscript.

\section{Additional information}

Supplementary information accompanies this paper at http://www.nature.com/ scientificreports

Competing financial interests: The authors declare no competing financial interests. 
How to cite this article: Yang, D.C., Carpena-Núñez, J., Fonseca, L.F., Biaggi-Labiosa, A. \& Hunter, G.W. Shape-controlled synthesis of palladium and copper superlattice nanowires for high-stability hydrogen sensors. Sci. Rep. 4, 3773; DOI:10.1038/srep03773 (2014). (c) (1) () () This work is licensed under a Creative Commons Attribution列 visit http://creativecommons.org/licenses/by-nc-nd/3.0 